19. As an example of a question in cubic carves to which the condition (22) may be applied, suppose it be required

"To find the locus of a point (P) such that, when tangents are drawn from it touching $a$ cissoid in $T_{1}, T_{2}, T_{3}$, and meeting the same curve again in $t_{1}, t_{2}, t_{3}$, the pencil joining the cusp (0) with these six points may be in involution, each of the three lines $O\left(T_{1} T_{2} T_{3}\right)$ corresponding to one of the three $O\left(t_{1} t_{2} t_{3}\right)$."

The Cissoid being $x^{3}-y^{2} z=0$, the equation to $O\left(T_{1}, T_{2}, T_{3}\right)$ is [Proceedings, Vol. 1I., p. $162(8)$ ], $x^{\prime} y^{\prime} z^{\prime}$ being the point $\mathrm{P}$,

$$
z^{\prime} y^{3}-3 x^{\prime} x^{2} y+2 y^{\prime} x^{3}=0
$$

that to the three lines $O\left(t_{1} t_{2} t_{3}\right)$ [ib. p. 164 (17)]

$$
4 z^{\prime} y^{3}-3 x^{\prime} x^{2} y-y^{\prime} x^{3}=0 \text {. }
$$

Here $\quad \Delta=4 z^{\prime}\left(y^{\prime \prime} z^{\prime}-x^{\prime 3}\right), \quad \Delta^{\prime}=16 z^{\prime}\left(y^{\prime 2} z^{\prime}-x^{\prime 3}\right)$;

$\theta$ reduces to $a d\left(a d^{\prime}+a^{\prime} d\right)+2 c^{2}\left(3 a c^{\prime}+a^{\prime} c\right)$, when $b=0, l^{\prime}=0$;

and $\theta^{\prime} \quad$ " $\quad a^{\prime} d^{\prime}\left(a d^{\prime}+a^{\prime} d\right)+2 c^{\prime 2}\left(a c^{\prime}+3 a^{\prime} c\right), \quad " \quad " \quad "$

so that here $\theta=14 z^{\prime}\left(x^{3}-y^{\prime 2} z^{\prime}\right), \theta^{\prime}=2 z^{\prime}\left(13 x^{\prime 3}+14 y^{\prime 2} z^{\prime}\right)$;

and $\quad \Delta \theta^{\prime 2}-\Delta^{\prime} \theta^{3}=432 x^{\prime 3} z^{\prime 3}\left(x^{\prime 3}-y^{\prime 2} z^{\prime}\right)\left(x^{\prime 3}-28 y^{\prime 2} z^{\prime}\right)$.

Rejecting the irrelevant factors, the required locus is therefore

$$
x^{3}-28 y^{2} z=0 \text {, }
$$

a curve generated from the cissoid by dividing its ordinates in a constant ratio.

The problem of finding the envelopo of a transversal cutting two cubic curves in six points in involution, again, would bo solved by substituting in them for $z$ from the equation to the transversal. Applying the condition (22) to the two resulting cubics in $x$ and $y$, the result would be the tangential equation to the required envelope, which would probably be of the 18th class.

Mr. W. K. Clifford read the following :-

\title{
On a Caso of Evaporation in the Order of a Resultant.
}

A particular case of the following theorem was required in the conrso of $\mathrm{my}$ proof that every rational equation has a root; but I have thought that the theorom itself (though indced a mere obvious remark) was worthy of being placed on record, because of the extremely small number of results of this kind that have yet been arrived at, and of their great importance in analysis.

I'scorcuc. Ixt it be required to eliminate $a$ betwecn two equations homogeneous in $x$ and certain other variables $y, z, \ldots$, in which equations, however, $x$ only occurs in virtue of the occurrence of a quantity $w=x^{n} y^{\beta} z^{\gamma} . . .$, where $\alpha+\beta+\gamma+\ldots=\mu$; let also $m, n$ be the orders of 
the equations, and $h, k$ the remainder's after division of $m, n$ respectively by $\mu$; then the order of the resultant is $=\frac{m n-h k}{\mu}$.

Demonstration. Suppose that $p, q$ are the quotients of the division of $m, n$ respectively by $\mu$; that is to say, let

then the two equations may be written

$$
m=p \mu+h, n=q \mu+k,
$$

$$
\begin{aligned}
& a_{m}+a_{m-\mu} \cdot w+a_{m-2 \mu} \cdot w^{2}+\ldots+a_{h} w^{p}=0, \\
& b_{n}+b_{n-\mu} \cdot w+b_{n-2 \mu} \cdot w^{2}+\ldots+b_{k} w^{q}=0 ;
\end{aligned}
$$

where the suffixes of the several coefficients indicate their orders in the variables $y, z, \ldots$ Instead of directly eliminating $x$ from these equations, we may eliminate $w$; and the result of this may be written down at once by Professor Sylvester's dialytic method. It is in fact

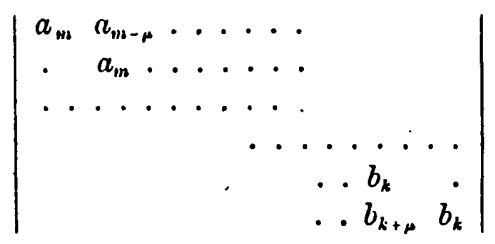

where in the principal diagonal of the determinant the constituent $a_{m}$ occurs $q$ times, and the constituent $b_{k}$ occurs $p$ times. The order of the resulting term $\left(a_{m}\right)^{q} \cdot\left(b_{k}\right)^{p}$ is $m q+k p$, and this therefore (since the determinant must be homogeneous) is the order of the resultant itself. If we had written the $b$ coefficients before the $a$ coefficients, we should bave obtained $u p+h q$ as the value of the same quantity. These two values are identical, since by hypothesis

$$
q(n-h)=p(n-k)=p q \mu,
$$

and therefore $q n+p l i=p n+q h=r$, suppose.

Now

$$
\begin{aligned}
m n & =(p \mu+h)(q \mu+k), \\
& =p q \mu^{2}+p k \mu+h q \mu+h l, \\
& =\mu r+h l \\
\therefore \quad r & =\frac{m n-h k}{\mu},
\end{aligned}
$$

as was to be proved.

'The following extension is brought to light by a different methud of proving the original theorem.

Isut it be required to climinate $k-1$ variables $x, y, \ldots$ from $k$ cquantions homogeneous in theso and ceriain other variables, in which equations, however, $x, y, \ldots$ only occur in virtue of the occurrence of $k-1$ quantities $u, v, \ldots$ all of the same order $\mu$; let also $m_{1}, m_{2}, \ldots u_{\downarrow}$ lo the orders of tho equations, and

voL. H1. No. 26 .

$$
m_{i}=l_{i} \mu+h_{i}, \quad h_{i}<\mu ;
$$


then the order of the resultant is

For the equations may be written

$$
\Pi_{p}\left(\Sigma \frac{h}{p}+\mu\right)
$$

$$
a_{h}(u, v, \ldots)^{p}+\theta a_{h+\mu}(u, v, \ldots)^{-1}+\ldots+\theta^{p} a_{h+p \mu}=0,
$$

where the $h, p$ are to be affected successively with the suffixes $1,2, \ldots k$, and $\theta$ may be considered $=1$. Now these equations may be regarded as having coefficients of the constant order $h$, but the weight of every coefficient of $\theta^{r}$ equal to $r \mu$. This being so, the degree of the resultant in the neliminated variables will be the sum of its order and weight calculated on these suppositions. But its order is $h_{1} p_{2} p_{3} \ldots p_{k}$, or $\frac{h_{1}}{p_{1}} \Pi_{p}$, due to the coefficients of the first equation, $\frac{h_{2}}{p_{2}} \Pi_{p}$ due to the coefficients of the second, and so on; while its weight is $\mu \Pi p$. Hence the entire order of the resultant is

as stated above.

$$
\Pi_{p}\left(\Sigma \frac{h}{p}+\mu\right)
$$

NFarch 10th, 1870.

Prof. CAYLEY, President, in the Chair.

Mr. E. A. L. Bradshaw Smith was elected a Member, and Micssrs. A. and W. M. Ramsay were admitted into the Society. Visitor, Prof. Oppermann of Copenhagen.

Mr. Tucker read two communications by Mr. Clerk-Maxwell: the one on "Topographical Geometry" (on which papor the President aud Mr. Archibald Smith made some remarks); the other

On the Displacement in a Case of Fluid Motion.

In most investigations of fluid motion, we consider the velocity at any point of the fluid as defined by its magnitude and direction, as a function of the coordinates of the point and of the time. We are supposed to be able to take a momentary glance at the system at any time, and to observe the velocities; but are not required to be ablo to keep our eyo on a particular molccule during its motion. This method, thercfore, properly belongs to the thenry of a contiuuous fluid alike in all its parts, in which we measure the velocity by the volume which passes through unit of area rather than by the distance travelled by a molecule in unit of time. It is also the only method applicable to tho case of a fluid, the motions of the individual molecules of which are not expressible as functions of their position, as in the motions due to heat and diffusion. When similar equations occur in the theory of the con- 